\title{
DISCUSSION
}

\section{Lateral spreading within a limit equilibrium framework: Newmark's sliding blocks with degrading yield accelerations}

\author{
BEN A. LESHCHINSKY*, H. BENJAMIN MASON†, MICHAEL J. OLSEN $\dagger$, DANIEL T. GILLINS $\$$, \\ SANJAY KUMAR SHUKLA $\S$ and PRAGYAN PRADATTA SAHOO\|
}

\section{Contribution by Sanjay Kumar Shukla and Pragyan Pradatta Sahoo}

The authors have presented an important contribution to the lateral spreading behaviour of slope comprising a column of sliding blocks with degrading yield accelerations by using the modified Newmark's sliding block concept (Leshchinsky et al., 2018). The work presented is primarily concerned with the computation of lateral spreading displacement of the soil block throughout the soil profiles along with the shear strains and simplified earth pressures on the soil block. The proposed limit equilibrium approach evaluates the yield accelerations of multiple, thin, rigid slices within the soil block considering different forces in terms of slice weight, lateral earth pressure resultants, shear resistance, pseudostatic seismic force and boundary neutral forces (pore water pressures) in a time-dependent framework. However, in order to evaluate the displacement of each slice of the soil block, the time-dependent yield acceleration is an important part of any method for the assessment of the slope during the earthquake period. Although the authors considered both horizontal and vertical seismic coefficients in the equilibrium equations (equations (1) and (2)), the effect of the vertical seismic coefficient is missing in the expression of yield seismic acceleration (equation (15)), and consequently, the displacement of the slope in the expression is based solely on the effect of the horizontal seismic coefficient. Therefore, the discussers would like to add a revised form of the expression (15) of the original paper, where the effect of the vertical seismic coefficient has been neglected.

Readers should note that the derivation of equations (1) and (2), and further equations (12) and (15) are based on the force equilibrium of the $n$th slice of the liquefied soil layer having the base of the slice parallel to the slope surface rather than the whole soil block. Therefore, the discussers recommend the following notation for the $n$th slice, for the convenience of the readers.

$k_{\mathrm{h}: n}$ as $k_{\mathrm{h} n}$ and $k_{\mathrm{v}: n}$ as $k_{\mathrm{v} n}$ : seismic coefficients

$P_{\mathrm{L}: n}$ as $P_{\mathrm{L} n}$ and $P_{\mathrm{R}: n}$ as $P_{\mathrm{R} n}$ : earth pressure resultants

$U_{\mathrm{L}: n}$ as $U_{\mathrm{L} n}$ and $U_{\mathrm{R}: n}$ as $U_{\mathrm{R} n}$ : hydrostatic and excess pore water pressure resultants

$U_{\mathrm{b}: n}$ as $U_{\mathrm{b} n}$ and $U_{\mathrm{b}: n \text {-excess }}$ as $U_{\mathrm{b} n \text {-excess }}$ : basal hydrostatic and excess pore water pressure resultants

\footnotetext{
* Department of Forest Engineering, Resources and Management \& School of Civil and Construction Engineering, Oregon State University, Corvallis, OR, USA.

$\dagger$ School of Civil and Construction Engineering, Oregon State University, Corvallis, OR, USA.

* Formerly Oregon State University; now NOAA/National Geodetic Survey (N/NGS4), Silver Spring, MD, USA.

$\S$ Edith Cowan University, Joondalup, WA, Australia (Orcid:0000-0002-4685-5560).

|| Edith Cowan University, Joondalup, WA, Australia

(Orcid:0000-0002-6788-0415).
}

Some notation, which was defined in the context of a soil block subjected to different forces, is misleading in a few cases. For example, when a trapezoidal sliding block of weight $W$ is considered, the lateral boundary hydraulic resultant forces $U_{1}$ and $U_{2}$ on p. 701 should be replaced by $U_{\mathrm{L}}$ and $U_{\mathrm{R}}$, respectively, as correctly listed in the Notation list. Similarly, both symbols $k_{\mathrm{h}: n}$ and $k_{\mathrm{H}: n}$ have been used inconsistently in the equations. In equations (1) and (2), the notation for horizontal $\left(k_{\mathrm{h}: n}\right)$ and vertical $\left(k_{\mathrm{v}: n}\right)$ seismic coefficients was changed to $k_{\mathrm{H}: n}$ and $k_{\mathrm{V}: n}$ in equation (6). In the discussers' opinion, both $k_{\mathrm{h}: n}$ and $k_{\mathrm{H}: n}$ refer to the horizontal seismic coefficient. In a similar way, $k_{\mathrm{v}: n}$ and $k_{\mathrm{V}: n}$ as used refer to the vertical seismic coefficient. The symbol $k_{\mathrm{y}}$ has been defined as the yield acceleration, but it is the yield seismic coefficient which is a dimensionless parameter. Also, the notation for the weight $W_{1}$ in equation (3) should be considered $W$ as per the description provided earlier. While reading the paper, readers should note these key points.

From Fig. 15, considering the pseudo-static forces parallel and perpendicular to the basal, liquefied slip surface, using the aforementioned notations, the static equilibrium of the $n$th slice having depths $H_{\mathrm{L} n}$ and $H_{\mathrm{R} n}$ results in

$$
\begin{aligned}
\sum F_{\mathrm{II}}= & =\left(P_{\mathrm{L} n}-P_{\mathrm{R} n}\right) \cos \beta+\left(U_{\mathrm{L} n}-U_{\mathrm{R} n}\right) \cos \beta \\
& +k_{\mathrm{h} n} W_{n} \cos \beta+W_{n} \sin \beta-k_{\mathrm{v} n} W_{n} \sin \beta-T_{n}
\end{aligned}
$$

or

$$
\begin{aligned}
T_{n}= & \left(P_{\mathrm{L} n}-P_{\mathrm{R} n}\right) \cos \beta+\left(U_{\mathrm{L} n}-U_{\mathrm{R} n}\right) \\
& \times \cos \beta+\left[k_{\mathrm{h} n} \cos \beta+\left(1-k_{\mathrm{V} n}\right) \sin \beta\right] W_{n}
\end{aligned}
$$

and

$$
\begin{aligned}
\sum F_{\perp}= & =N_{n}+U_{\mathrm{b} n}+U_{\mathrm{b} n \text {-excess }}+k_{\mathrm{h} n} W_{n} \sin \beta \\
& +k_{\mathrm{v} n} W_{n} \cos \beta-\left(P_{\mathrm{L} n}+P_{\mathrm{R} n}\right) \sin \beta \\
& -\left(U_{\mathrm{L} n}+U_{\mathrm{R} n}\right) \sin \beta-W_{n} \cos \beta
\end{aligned}
$$

or

$$
\begin{aligned}
N_{n}= & {\left[\left(P_{\mathrm{L} n}+P_{\mathrm{R} n}\right)+\left(U_{\mathrm{L} n}+U_{\mathrm{R} n}\right)\right] \sin \beta } \\
& -\left(U_{\mathrm{b} n}+U_{\mathrm{b} n \text {-excess }}\right)+\left[\left(1-k_{\mathrm{v} n}\right) \cos \beta-k_{\mathrm{h} n} \sin \beta\right] W_{n}
\end{aligned}
$$

It may be noted that the components of forces $P_{\mathrm{R} n}$ and $U_{\mathrm{R} n}$, perpendicular to the slope, have been taken incorrectly, resulting in an error in the original equation (2) of the paper, compared to equation (17). The discussers expect that this error may affect the results as well as the graphical presentations, as discussed in the paper, to some extent.

Further, equation (7) in the original paper for the mobilised shear stress, contains $\sigma_{n}^{\prime}$ twice, which seems to be a typographical error. Equation (7) can be presented as

$$
\tau_{n-\mathrm{mob}}=\frac{c_{n}^{\prime}+\sigma_{n}^{\prime} \tan \phi_{n}^{\prime}}{\mathrm{FS}}
$$




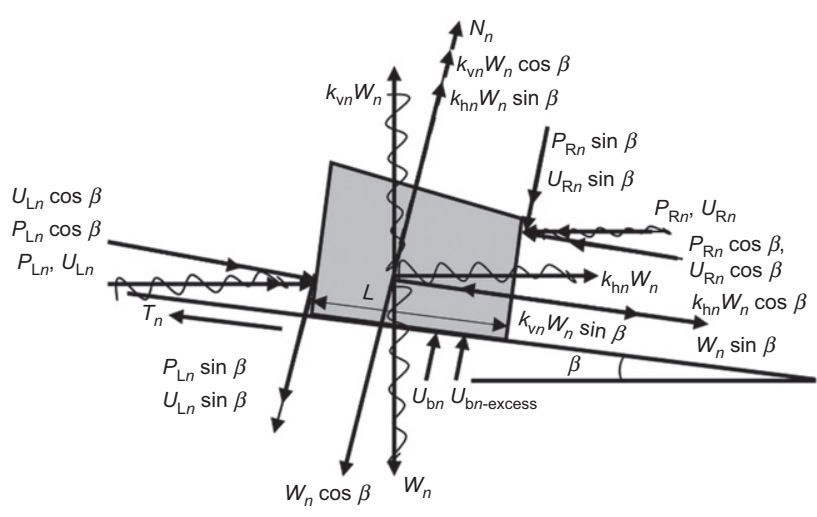

Fig. 15. Free-body diagram of the $n$th sliding slice

or

$$
\mathrm{FS}=\frac{c_{n}^{\prime}+\sigma_{n}^{\prime} \tan \phi_{n}^{\prime}}{\tau_{n-\mathrm{mob}}}
$$

or

$$
\mathrm{FS}=\frac{c_{n}^{\prime} L+\sigma_{n}^{\prime} L \tan \phi_{n}^{\prime}}{\tau_{n-\mathrm{mob}} L}=\frac{c_{n}^{\prime} L+N_{n} \tan \phi_{n}^{\prime}}{T_{n}}
$$

where $\sigma_{n}^{\prime} L=N_{n}$ and $\tau_{n \text {-mob }} L=T_{n}$.

Using equations (16) and (17) into (18), the factor of safety (FS) for the $n$th slice is expressed as clarification regarding the notation presented in the original study.

The authors respectfully disagree regarding the discussers' proposed orientation of the components of lateral boundary forces presented in the original study (e.g. $P_{\mathrm{L}}$ and $U_{\mathrm{L}}, P_{\mathrm{R}}$ and $U_{\mathrm{R}}$ ). The authors suspect that the source of this disagreement may stem from different consideration of boundaries, such as vertical as opposed to perpendicular edges of the free-body diagrams presented. Specifically, for the proposed case, the lateral boundary force resultants $\left(P_{\mathrm{L}}\right.$ and $U_{\mathrm{L}}, P_{\mathrm{R}}$ and $U_{\mathrm{R}}$ ) have opposing perpendicular (i.e. vertical shear) components with respect to the failure plane (Fig. 1 in the original study; p. 701). In other words, the vertical shear components acting on the outside boundaries would not both be downward as shown in Fig. 15. Using the original conventions presented, the components on the left boundary would have a slight positive vertical shear effect with respect to the presumed failure plane, and the components on the right boundary would have a slight negative vertical shear effect. Thus, for vertical boundaries, the authors believe that the normal component was defined appropriately for the $n$th block in equation (11) of the original paper as

$$
\begin{aligned}
N_{n}= & W_{n} \cos \beta+\left(P_{\mathrm{R}: n}+U_{\mathrm{R}: n}\right) \sin \beta \\
& -U_{\mathrm{b}: n}-U_{\mathrm{b}: n \text {-excess }}-\left(P_{\mathrm{L}: n}+U_{\mathrm{L}: n}\right) \sin \beta \\
& -k_{\mathrm{v}: n} W_{n} \cos \beta-k_{\mathrm{h}: n} W_{n} \sin \beta
\end{aligned}
$$

Note that in equation (22), the component of the boundary forces acting perpendicular to the failure surfaces is a

$$
\mathrm{FS}=\frac{c_{n}^{\prime} L+\left[W_{n} \cos \beta+\left(P_{\mathrm{R} n}+U_{\mathrm{R} n}\right) \sin \beta-U_{\mathrm{b} n}-U_{\mathrm{b} n-\mathrm{excess}}+\left(P_{\mathrm{L} n}+U_{\mathrm{R} n}\right) \sin \beta-k_{\mathrm{v} n} W_{n} \cos \beta-k_{\mathrm{h} n} W_{n} \sin \beta\right] \tan \phi_{n}^{\prime}}{\left(P_{\mathrm{L} n}+U_{\mathrm{L} n}\right) \cos \beta-k_{\mathrm{v} n} W_{n} \sin \beta+k_{\mathrm{h} n} W_{n} \cos \beta+W_{n} \sin \beta-\left(P_{\mathrm{R} n}+U_{\mathrm{R} n}\right) \cos \beta_{n}}
$$

or

$$
\mathrm{FS}=\frac{c_{n}^{\prime} L+\left\{\left[\left(P_{\mathrm{L} n}+P_{\mathrm{R} n}\right)+\left(U_{\mathrm{L} n}+U_{\mathrm{R} n}\right)\right] \sin \beta-\left(U_{\mathrm{b} n}+U_{\mathrm{b} n \text {-excess }}\right)+\left[\left(1-k_{\mathrm{v} n}\right) \cos \beta-k_{\mathrm{h} n} \sin \beta\right] W_{n}\right\} \tan \phi_{n}^{\prime}}{\left[\left(P_{\mathrm{L} n}-P_{\mathrm{R} n}\right)+\left(U_{\mathrm{L} n}-U_{\mathrm{R} n}\right)\right] \cos \beta+\left[k_{\mathrm{h} n} \cos \beta+\left(1-k_{\mathrm{v} n}\right) \sin \beta\right] W_{n}}
$$

When $\mathrm{FS}=1$, the yield seismic coefficient $k_{\mathrm{h} n \text {-yield }}(t)$ of the $n$th slice can be obtained from equation (20) as function of $\sin (\beta)$. Because most liquefaction-induced lateral spreads occur on very gently sloping ground (Keefer,

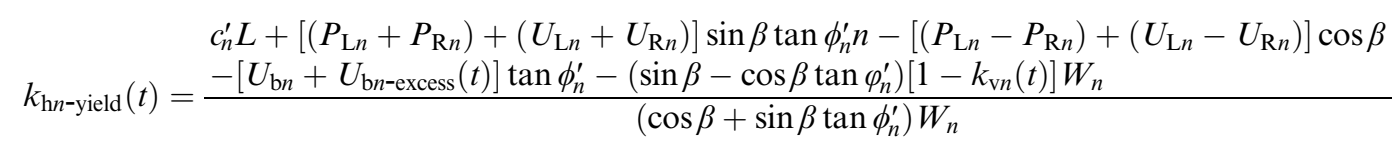

From equation (21), it can be noted that $k_{\mathrm{h} n \text {-yield }}(t)$ is not only the function of time and depth, but it also depends upon the coefficient of vertical seismic coefficient $k_{\mathrm{v} n}(t)$, which was ignored in equation (15) of the paper. This may lead to an error in seismic displacement of the slope, as it was calculated considering only the effect of the horizontal seismic coefficient.

\section{Authors' reply}

The authors appreciate the commentary from the discussers, their suggestions for considering vertical seismic thrust in the expression for yield acceleration, as well as
1984), $\beta$ may be only a few degrees, or even horizontal in some scenarios. Accordingly, $\sin (\beta)$ is negligible, or small at best, especially in comparison to the already significant uncertainties associated with characterising the onset of liquefaction and ground deformations under seismic excitation. In the authors' proposed framework, the largest drivers of lateral spreading in the given example are $(a)$ excess pore pressure, $(b)$ horizontal, inertial loading owing to seismicity and (c) changes in lateral stress that occur from changes in surface topography and confinement (Youd et al., 2002).

As the discussers correctly stated, the authors only considered horizontal accelerations in the original study to match their theoretical results with experimental centrifuge 
data where only horizontal seismic excitation was applied. The authors also recognise that earthquake-induced liquefaction, which can lead to lateral spreading, initiates at some depth under the ground surface. At the liquefaction initiation depth, the ground shaking is dominated by body waves, and primarily vertically propagating shear waves, which impart a horizontal acceleration. Accordingly, the authors also think that their simplification of only considering horizontal accelerations is justified for many cases, and they note that the importance of vertical acceleration on seismic slope stability has been a source of debate (Ling \& Leshchinsky, 1998; Matasovic et al., 1998; Ingles et al., 2006). Notwithstanding the foregoing arguments, if significant vertical accelerations are present, then they directly influence effective stress, which affects yield accelerations as well. Therefore, in field applications where vertical excitations may be present, the authors agree that considering vertical acceleration is worthwhile. Based on the conventions reported in the authors' original study, a revised expression for yield acceleration considering vertical accelerations is

\section{REFERENCES}

Ingles, J., Darrozes, J. \& Soula, J. C. (2006). Effects of the vertical component of ground shaking on earthquake-induced landslide displacements using generalized Newmark analysis. Engng Geol. 86, No. 2-3, 134-147.

Keefer, D. K. (1984). Landslides caused by earthquakes. Geol. Soc. Am. Bull. 95, No. 4, 406-421.

Leshchinsky, B. A., Mason, H. B., Olsen, M. J. \& Gillins, D. T. (2018). Lateral spreading within a limit equilibrium framework: Newmark sliding blocks with degrading yield accelerations. Géotechnique 68, No. 8, 699-712, https://doi.org/10.1680/jgeot. 16.P.292.

Ling, H. I. \& Leshchinsky, D. (1998). Effects of vertical acceleration on seismic design of geosynthetic-reinforced soil structures. Géotechnique 48, No. 3, 347-373, https://doi.org/10.1680/geot. 1998.48.3.347.

Matasovic, N., Kavazanjian, E. Jr \& Giroud, J. P. (1998). Newmark seismic deformation analysis for geosynthetic covers. Geosynthetics Int. 5, No. 1-2, 237-264.

Youd, T. L., Hansen, C. M. \& Bartlett, S. F. (2002). Revised multilinear regression equations for prediction of lateral spread displacement. J. Geotech. Geoenviron. Engng 128, No. 12, 1007-1017.

$$
k_{\mathrm{H}: n \text {-yield }}(t)=\frac{\begin{array}{c}
c_{n}^{\prime} L+\left\{\left[1-K_{\mathrm{v}}(t)\right] W_{n} \cos \beta+\left(P_{\mathrm{R}: n}+U_{\mathrm{R}: n}\right) \sin \beta-U_{\mathrm{b}: n}-U_{\mathrm{b}: n \text {-excess }}(t)-\left(P_{\mathrm{L}: n}+U_{\mathrm{L}: n}\right) \sin \beta\right\} \tan \phi_{n}^{\prime} \\
-\left(P_{\mathrm{L}: n}+U_{\mathrm{L}: n}\right) \cos \beta+\left(P_{\mathrm{R}: n}+U_{\mathrm{R}: n}\right) \cos \beta-\left[1-k_{\mathrm{v}}(t)\right] W_{n} \sin \beta
\end{array}}{W_{n} \sin \beta \tan \phi_{n}^{\prime}+W_{n} \cos \beta}
$$

Once again, the authors thank the discussers for their interest, their clarifications as regards notation and their suggestions for improving the authors' original study. 